Телекоммуникационные системы

и компьютерные сети

УДК 621.397

DOI 10.5862/JCSTCS.212.1

В.Ю. Бабков, А.В. Никитина, В.В. Стариков

ОПРЕДЕЛЕНИЕ ПРОСТРАНСТВЕННО-ТЕХНИЧЕСКИХ ПАРАМЕТРОВ
СОТОВОЙ СЕТИ СТАНДАРТА LТЕ

V.Yu. Babkov, A.V. Nikitina, V.V. Starikov

\title{
DEFINITION OF THE SPATIAL AND TECHNICAL PARAMETERS LTE NETWORK
}

\begin{abstract}
Предложена методика построения сети начального приближения стандарта LTE. Изложены основы метода выбора кластерной структуры и экспресс-анализа сетевой конфигурации на соответствие требуемым параметрам по емкости и пропускной способности сети.

ПЛАНИРОВАНИЕ СТАНДАРТОВ СВЯЗИ ЧЕТВЕРТОГО ПОКОЛЕНИЯ; ЧАСТОТНЫЙ КЛАСТЕР; ПРОПУСКНАЯ СПОСОБНОСТЬ; АБОНЕНТСКАЯ ЕМКОСТЬ; БЮДЖЕТ ПОТЕРЬ.

This article contains methods of planning the initial approximation LTE network and choosing the cluster structure. All main problems of RF Planning are inside the initial approximation LTE network. This network requires the accurate calculation of capacity, subscriber capacity and cluster structure. We have offered the system of mass service for calculating the subscriber capacity. Spatial and technical parameters need clarification too. They will depend on MAPL when using the selected MCS and equipment parameters. Therefore the planning procedure can be started only after defining these parameters. This article presents the algorithm for generating the initial approximation LTE network.
\end{abstract}

LTE RF PLANNING; CLUSTER 4G; LTE RADIO LINK BUDGET.

История сотовых систем мобильной радиосвязи насчитывает уже несколько десятилетий. Разработаны общие принципы построения сетей мобильной радиосвязи, включая подготовку исходных данных, построение начального приближения и структурно-параметрическую оптимизацию [1, 2]. Решение задачи построения сети мобильной связи стандарта LTE предполагает использование метода приближений в соответствии с алгоритмом частотнотерриториального планирования [2]. Алгоритм раскрывает последовательность и содержание этапов построения начального приближения и итеративной оптимизации сети при широком использовании средств программного обеспечения, поддерживающих функции синтеза сети и анализа экс- плуатационных характеристик.

Следует отметить, что на сегодняшний день вопросы частотно-территориального планирования в сетях мобильной связи стандарта LTE являются наименее разработанными и требуют дальнейшего исследования. В частности, важно решение задачи начального приближения, когда вся сеть декомпозируется на однородные фрагменты, применительно к которым находятся распределения базовых станций по зонам обслуживания, параметры базовой сети и распределение частотного ресурса. Планирование начального приближения сети мобильной связи стандарта LTE имеет много общего с планированием сетей мобильной связи стандартов $2 \mathrm{G} / 3 \mathrm{G}$, поскольку также представляет собой пошаговое определение 
ее пространственно-технических параметров. В то же время построение начального приближения для большинства стандартов сотовых сетей $2 \mathrm{G} / 3 \mathrm{G}$ осушествляется для «тотальной» услуги, которой является передача речи. Найденная в процессе планирования сеть мобильной радиосвязи будет решением, относительно которого проводятся оценки по зоне покрытия, абонентской емкости, качеству радиосвязи, скорости передачи, пропускной способности и т. п. для других видов услуг. В отличие от сетей $2 \mathrm{G} / 3 \mathrm{G}$ сети мобильной связи стандарта LTE являются сетями передачи пакетного трафика, а качество услуг на этапе планирования сети оценивается доступной для пользователей скоростью передачи данных, допустимым временем и надежностью доставки пакетов данных, которые зависят от состояния канала радиосвязи, помеховой обстановки и параметров трафика.

В настоящей статье в постановочном плане обсуждаются именно эти вопросы применительно к задаче построения сети начального приближения.

Выбор типа частотного кластера. Построение сети LTE целесообразно осуществлять на основе частотных кластеров, представляющих собой группу примыкающих друг к другу сот, в которой при использовании всего частотного ресурса сети однозначно может быть решена задача достижения максимальной емкости сети при соблюдении приемлемого уровня внутрисистемных помех. В случае успешного решения задачи по формированию такой группы сот, ее можно использовать как некоторую минимальную структурную единицу при планировании сети LTE. Сеть начального приближения будет строиться путем повтора одних и тех же частотных кластеров в пределах зоны обслуживания. Конфигурация частотного кластера будет непосредственно влиять на такие важнейшие параметры системы, как уровень внутрисистемных помех и реализуемая сетевая емкость. При этом значительно упрощается задача увеличения емкости сети, т. к. каждый частотный кластер сети, отвечающий требованиям по уровню внутрисистемных помех, является оптимальным по этому параметру.

Частотный кластер выбирается исходя из ожидаемой абонентской нагрузки и значения вероятности появления внутрисистемных помех $P(C)$, где $C$ - размерность частотного кластера. Методика вычисления вероятности $P(C)$ описана в [1]. В качестве примера на рис. 1 приведен фрагмент несекторированного частотного кластера с дробным повторным назначением частот. Вся полоса частот, выделенная системе, состоит из четырех равных полос f1, f2, f3 и f4.

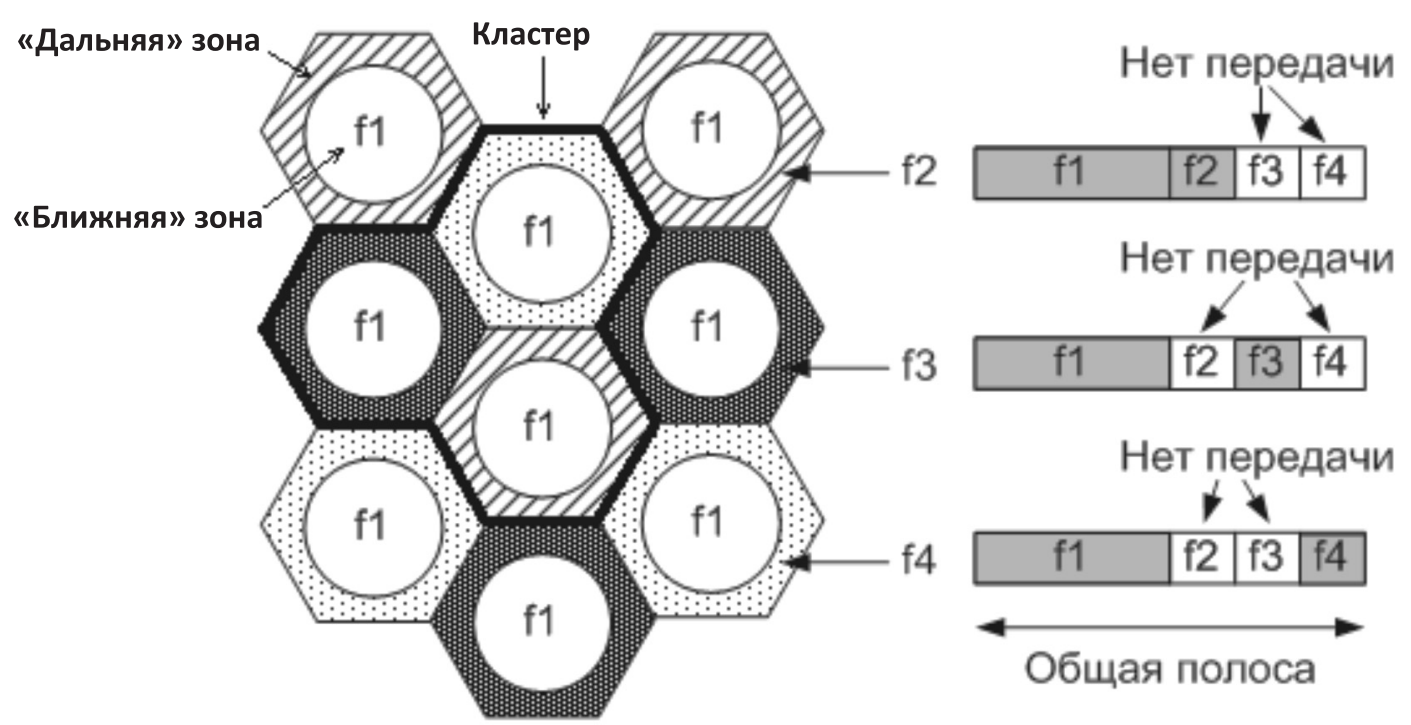

Рис. 1. Фрагмент сети на основе несекторированного кластера размером $C=3$ с дробным повторным назначением частот 
При такой структуре каждая базовая станция, входящая в кластер, использует до $50 \%$ частотного ресурса сети при полном использовании всей полосы частот в кластере. Подробно процедура построения частотного кластера в сетях LTE при дробном и мягком назначении частот рассмотрена в [2].

Основной сложностью при построении структуры частотного кластера при заданной вероятности появления внутрисистемных помех является максимизация емкости и пропускной способности кластерной структуры, исходя из доступного частотного ресурса сети. Эта задача, по состоянию на сегодняшний день, требует дополнительного исследования.

Определение пространственных параметров сети. В процессе определения пространственных параметров сети производится оценка бюджетов потерь, радиусов «ближней» и «дальней» зон, определение мощностей передатчиков для «ближней» и «дальней» зон и определение числа БС в однородной сети. Бюджет потерь определяет максимально-допустимые потери на трассе распространения радиоволн (MAPL) для конкретного вида сигнально-кодовой конструкции при заданных параметрах приемо-передающего оборудования сети. Бюджет потерь не зависит от выбранной модели потерь распространения сигнала, но должен учитывать направление связи по линии «вверх» и линии «вниз», поскольку в сотовых системах мощность передатчика абонентской станции обычно меньше мощности передатчика базовой станции. Результаты оценок бюджета потерь [2] при различных конфигурациях оборудования и используемых сигнально-кодовых конструкций показывают, что ограничивающей по дальности связи является линия «вверх», т. к. мобильная станция, в отличие от базовой, обладает меньшей мощностью передатчика и имеет ненаправленную антенну. При этом допустимые потери при увеличении полосы рабочих частот системы падают, и для сохранения энергетического баланса между каналами необходимо ограничивать количество ресурсных блоков, приходящихся на абонентскую станцию: при полосе частот канала 10 МГц допустимые потери находятся в пределах 125,8-148 дБ и целесообразно ограничивать количество ресурсных блоков, выделяемых абонентской станции, до четырех;

при ширине полосы частот канала 15 МГц допустимые потери находятся в пределах 121,8-144,2 дБ и целесообразно ограничивать количество ресурсных блоков, выделяемых абонентской станции, до восьми;

при ширине полосы частот канала 20 МГц допустимые потери находятся в пределах 117,8-139,9 дБ и целесообразно ограничивать количество ресурсных блоков, выделяемых абонентской станции, до 16.

Чувствительности приемников и уровни максимально допустимых потерь на линии радиосвязи связаны с типом модуляции (видом используемой сигнально-кодовой конструкции) и полосой частот, занимаемой системой. Увеличение полосы частот в два раза (с 10 до 20 МГц) приводит к ухудшению чувствительности на 3-5 дБ и уменьшению максимально допустимых потерь на трассе радиосвязи (бюджета потерь) на 6-8 дБ.

Оценка бюджета потерь начинается с конкретизации требуемого (допустимого) отношения с/ш на входе приемников в линиях «вверх» и «вниз». Значения отношения с/ш в зависимости от используемой модуляционно-кодирующей схемы (MCS), условий приема, уровня сетевой загрузки и количества приемных антенн приведены в таблице [3, 4].

Полученное значение MAPL используется в дальнейшем для нахождения максимального радиуса соты (максимально возможного расстояния между приемником и передатчиком).

При планировании сети окончательное значение MAPL определяется с учетом запасов на интерференцию, затенение и проникновение в здание/автомобиль. Запас на интерференцию зависит от числа повторно используемых частот и загрузки в соте [2]. Запас на затенение учитывает затухание сигнала вследствие движения абонентского терминала, т. к. при этом часто теряется прямая видимость между абонентской и базовой станциями. Этот запас гарантирует 


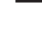

Требуемые значения с/ш в зависимости от MCS

\begin{tabular}{|c|c|c|c|}
\hline $\begin{array}{c}\text { Модуляционно-кодирующая } \\
\text { схема }\end{array}$ & $\begin{array}{c}\text { Условия } \\
\text { приема, Гц }\end{array}$ & $\begin{array}{c}\text { Сетевая } \\
\text { загрузка, \% }\end{array}$ & $\begin{array}{c}\text { Требуемое } \\
\text { значение с/ш, дБ }\end{array}$ \\
\hline \multirow{3}{*}{ QPSK 1/3 } & EPA, 5 & 30 & $-3,6$ \\
\hline & EVA, 5 & 30 & $-2,5$ \\
\hline & ETU, 70 & 30 & $-2,3$ \\
\hline \multirow{3}{*}{ QPSK 1/3 } & EPA, 5 & 70 & 0,3 \\
\hline & EVA, 5 & 70 & 1,8 \\
\hline & ETU, 70 & 70 & 2,1 \\
\hline \multirow{3}{*}{ 16-QAM 3/4 } & EPA, 5 & 30 & - \\
\hline & EVA, 5 & 30 & 5 \\
\hline & ETU, 70 & 30 & - \\
\hline \multirow{3}{*}{ 16-QAM 3/4 } & EPA, 5 & 70 & 11,6 \\
\hline & EVA, 5 & 70 & 12,4 \\
\hline & ETU, 70 & 70 & - \\
\hline 64-QAM 5/6 & - & 30 & - \\
\hline \multirow{3}{*}{ 64-QAM 5/6 } & EPA, 5 & 70 & 19,4 \\
\hline & EVA, 5 & 70 & 19,6 \\
\hline & ETU, 70 & 70 & - \\
\hline \multicolumn{4}{|c|}{$\begin{array}{l}\text { Варианты условий приема: } \\
\text { Extended Pedestrian A (EPA) - UE находится у пешехода; } \\
\text { Extended Vehicular A model (EVA) - UE находится внутри автомобиля; } \\
\text { Extended Typical Urban model (ETU) - стандартная модель городской застройки }\end{array}$} \\
\hline
\end{tabular}

процент локальных зон в пределах покрываемой территории, где уровень сигнала будет выше требуемого значения. Запас на проникновение применяется по отношению к зданиям, автомобилям и т. д., то есть к тем объектам, внутри которых необходимо произвести оценку уровня сигнала. Величина запаса будет зависеть от поглощающих свойств материала объекта, его конструктивных особенностей и рабочего диапазона частот системы связи. Как правило, запас на проникновение берут равным 8 дБ при использовании абонентской станции внутри автомобиля и 20 дБ и более при использовании в здании [1, 2].

Ниже в качестве примера представлены некоторые результаты анализа, в частности:

графики зависимости максимально допустимых потерь MAPL (дБ) и радиуса соты $R_{\text {соты max }}($ Км) при различных видах модуляции от числа ресурсных блоков $N_{R B}$ в диапазоне 2,6 ГГц (рис. 2, 3); зависимость максимального радиуса $R_{\text {соты max }}$ от используемой модуляционнокодирующей схемы (рис. 4) при работе сети в диапазоне 2600 МГц и выделенных полоcax 1,4 (6 РБ) и 20 МГц (100 РБ).

Результаты анализа показывают следующее:

- с ростом полосы частот, выраженной в количестве выделенных системе РБ, уменьшается MAPL и, соответственно, уменьшается максимально допустимый радиус соты;

- при работе сети в диапазоне частот 2600 МГц при 6 РБ и 100 РБ отношение $\frac{R_{\mathrm{QPSK} 1 / 3}}{R}$ примерно равно 3,5 и практиче$R_{64-\mathrm{QAM} 5 / 6}$

ски не меняется с уменьшением частоты связи.

Полученное значение бюджета потерь используется в дальнейшем для нахождения радиусов «ближней» и «дальней» $3 о н$. Задаваясь частотным диапазоном и количеством ресурсных блоков, можно оценить 

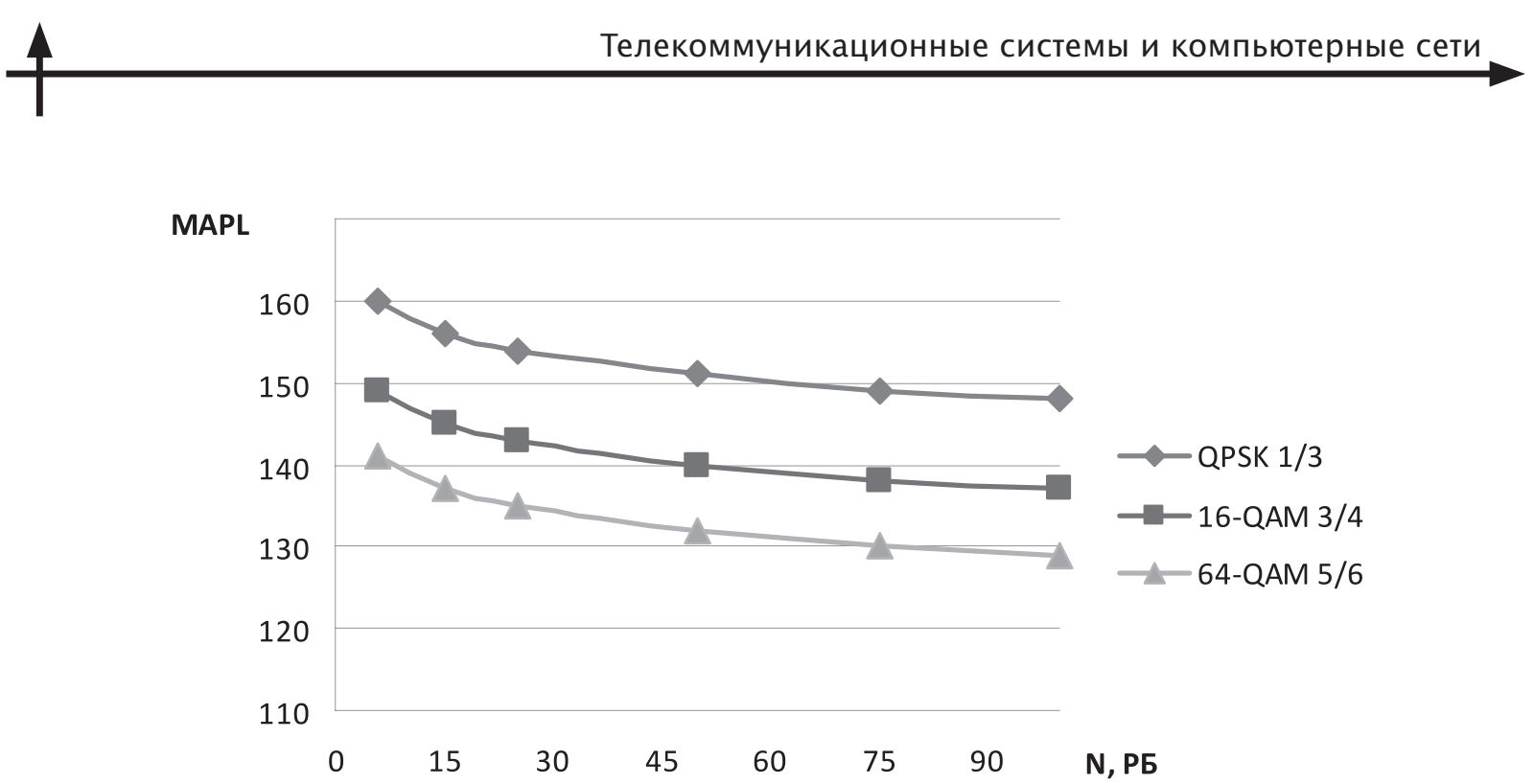

Рис. 2. Зависимость максимально допустимых потерь MAPL, дБ

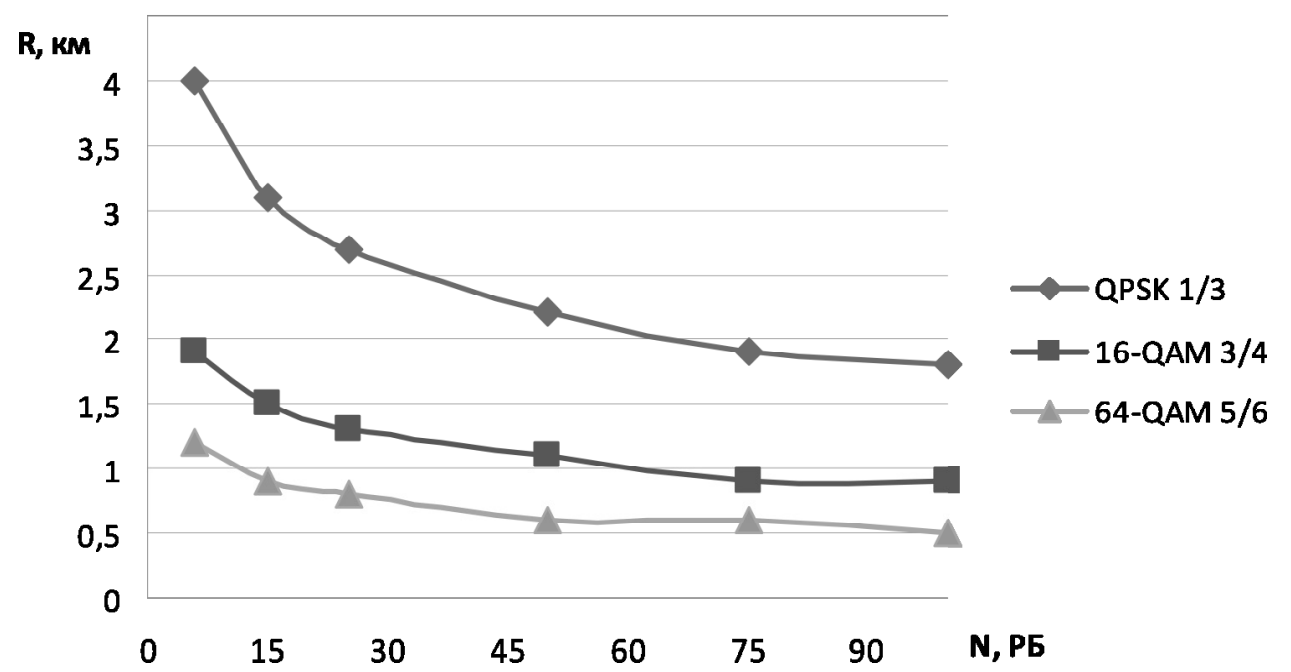

Рис. 3. Зависимость радиуса соты $R_{\text {соты max }}$ Км

для всего набора сигнально-кодовых конструкций распределение скоростей передачи в соте при различных условиях приема. При определении мощности передатчиков в «ближней» и в «дальней» зонах используются статистические и дифракционные модели расчета средних потерь на трассе распространения. Будем полагать, что отношение мощности излучения передатчика базовой станции в «ближней» зоне к мощности в «дальней» зоне пропорционально отношению

$$
\frac{P_{\text {изл.бз }}}{P_{\text {изл.дз }}}=\left(\frac{r}{R}\right)^{k},
$$

где $r$ - радиус «ближней» зоны соты; $R-$ радиус соты; $k$ - параметр, характеризующий условия радиосвязи ( $k=4$ в условиях города, 2 - в условиях открытой местности).

На основе полученного значения максимально допустимого радиуса соты производится расчет площади соты и находится число базовых станций в однородном фрагменте сети $N_{\text {Бс }}=\frac{S_{\text {сети }}}{S_{\text {соты }}}$, где $S_{\text {сети }}-$ площадь зоны обслуживания сети, $S_{\text {соты }}-$ площадь обслуживания базовой станции.

Таким образом, при определении пространственных параметров сети необходи- 

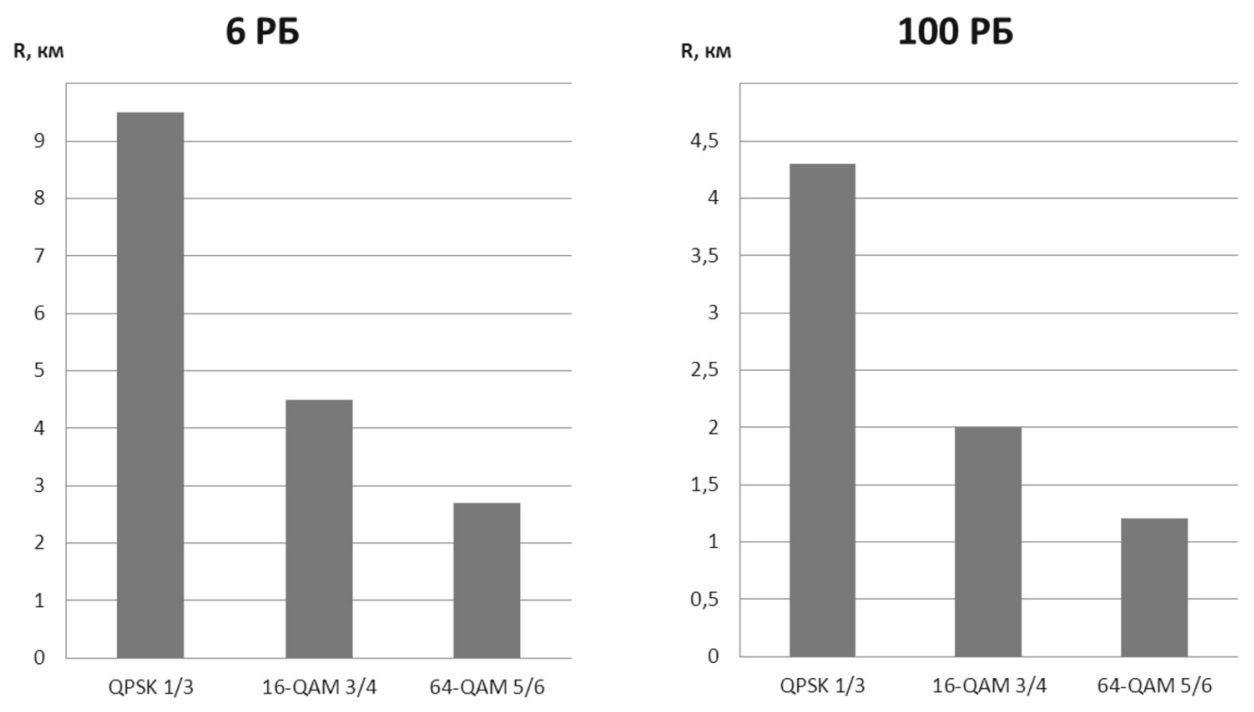

Рис. 4. Зависимость максимального радиуса $R_{\text {соты max }}$ от используемой модуляционно-кодирующей схемы

мо задаться частотным диапазоном, количеством ресурсных блоков, распределением скоростей передачи в соте, конфигурацией оборудования и условиями функционирования системы LTE.

Оценка средней пропускной способности и емкости. При оценке средней скорости передачи данных в прямом канале будем полагать, что распределение абонентских терминалов в зоне обслуживания базовых станций равномерно. Базовые станции предоставляют абонентам максимально возможную скорость передачи в соответствующих сегментах в «ближней» и «дальней» зонах [2, 5]. В общем случае средняя скорость передачи в «ближней» и «дальней» зонах в прямом канале находится по формуле

$$
V_{\mathrm{cp}}=\sum_{i=1}^{15} P_{i} V_{t x i},
$$

где $i$ - номер внутреннего сегмента в соответствующей зоне (для LTE $i=1 \ldots 15$ ); $P_{i}-$ вероятность нахождения абонента в $i$-сегменте соответствующей зоны; $V_{t x i}-$ скорость передачи, обеспечиваемая в i-сегменте соответствующей зоны.

Средняя скорость передачи в соте представляет сумму средних скоростей передачи в «ближней» и «дальней» зонах. Средняя скорость передачи данных зависит от распреде- ления абонентов в зонах обслуживания сети. В свою очередь, средняя пропускная способность сети зависит от алгоритма распределения ресурсов прямого канала (алгоритма работы планировщика). Как показывают исследования [2], алгоритм работы планировщика существенно влияет на параметры качества обслуживания. В сетях LTE вопрос выполнения заданной дисциплины обслуживания, например, учет приоритетности абонентов или абонентского трафика, предоставления ресурсов для абонента в том или ином объеме и др., возложен на планировщика сети. Планировщик представляет собой программный продукт, производящий в режиме реального времени управление частотно-временными ресурсами системы [2, 5]. Например, планировщик базовой станции может управлять ресурсом, исходя из алгоритма пропорциональносправедливого назначения ресурса. При таком алгоритме работы в каждом субкадре планировщик назначает приоритеты пользовательским трафиковым каналам и, следовательно, принимает решение о начале передачи трафика того или иного абонента.

Для обеспечения максимальной пропускной способности можно использовать алгоритм выделения ресурса на основе максимального показателя уровня помех. Данный алгоритм позволяет существенно 
повысить пропускную способность, т. к. учитывает показатель качества каждого канала и обслуживает в первую очередь пользователей с «хорошим» каналом. К основному недостатку этого алгоритма можно отнести негарантированное предоставление ресурсов при высокой нагрузке на сеть абонентам с низким соотношением с/ш. Если требуемая пропускная способность превышает допустимые значения, то необходимо перераспределение трафика между соседними базовыми станциями посредством эстафетной передачи абонентов, находящихся на границе зоны обслуживания.

Методика оценки числа равномерно распределенных по зоне обслуживания пользователей мобильного Интернета (трафик представляет собой пуассоновский поток) в установившемся режиме функционирования сети приведена в [2].

Вариант стандарта LTE, основанный на частотном дуплексе, использует симметричные полосы частот, предназначенные для передачи данных на линиях «вверх» и «вниз» соответственно. Учитывая тот факт, что в мобильных сетях передачи данных доля трафика на линии «вниз» составляет около $80 \%$ передаваемого в сети трафика (ситуация ассиметричного трафика), оценку потенциальных возможностей сети LTE по обслуживанию пользователей следует проводить с учетом количества абонентов, взятых на обслуживание по прямому каналу [2].

С учетом сложности профиля трафика системы LTE среднее время занятия канала одним абонентом $\bar{T}_{c}$ представляет собой величину, которая может быть получена только статистическим образом. При предварительном проектировании для получения оценочных расчетов следует задаться рядом значений $\bar{T}_{c}$ и уровнем загрузки базовой станции в ЧНН. Величину нагрузки можно определить, исходя из заданного уровня отказов в соединении. Для многоканальных СМО с ограниченной очередью длиной $m$ вероятность отказов определяется по формуле (см. например $[2,6])$

$$
P_{\text {отк }}=\frac{\rho^{n+m}}{n^{m} n !} P_{0} \text {, }
$$

где

$$
P_{0}=\left[1+\sum_{i=1}^{n} \frac{\rho^{n}}{n !}+\frac{\rho^{n+1}}{n * n !} \frac{1-\left(\frac{\rho}{n}\right)^{m}}{1-\frac{\rho}{n}}\right]^{-1}
$$

- вероятность того, что все каналы системы свободны; $n$ - число каналов СМO; $m-$ длина очереди; $\rho$ - нагрузка одного канала.

Задаваясь допустимым значением вероятности отказов, находим соответствующее ей значение $\rho=\frac{\lambda}{n} \bar{T}_{c}$, где $\lambda-$ поток заявок от всех абонентов в ЧНН. Число абонентов в зоне обслуживания можно определить KaK

$$
N_{\text {аб.Пк }}=\frac{n \rho}{\lambda_{1} \bar{T}_{c}},
$$

где $\lambda_{1}-$ поток заявок от одного абонента в ЧНН.

Например, если в системе значение $\rho=0,3$ при 100 ресурсных блоках (пусть на один канал трафика приходится один ресурсный блок), $\lambda_{1}=$ одной заявке в час и $\bar{T}_{c}=1 / 12$ ч, то число абонентов, взятых на обслуживание базовой станцией в ЧНН, равно 360 .

Очевидно, что задача оценки средней пропускной способности и емкости сети может быть решена для заданного профиля абонентского трафика и алгоритма работы планировщика, а в условиях трансформации трафика от пуассоновского потока к самоподобному требует отдельного исследования.

Частотное планирование. Частотное планирование включает решение следующих задач:

1) назначение полос частот секторам базовых станций;

2) распределение идентификаторов PCI (physical cell identity - физический идентификатор соты);

3) планирование преамбулы при случайном доступе в канале PRACH (Physical Random Access Channel - физический канал случайного доступа);

4) планирование пилотных сигналов. 
Назначение полос частот секторам базовых станций производится в соответствии с частотным кластером, использованным при построении однородного фрагмента сети по аналогии с частотным назначением в стандарте GSM. В стандарте LTE определено 504 уникальных идентификатора соты PCI, которые рассчитываются на основе первичного и вторичного сигналов синхронизации. Алгоритм применения P-SCH аналогичен механизму распределения скремблирующего кода в системах WCDMA [2]. Вторичный сигнал синхронизации является идентификатором соты. При планировании сети для базовых станций, зоны обслуживания которых находятся рядом или перекрываются, необходимо использовать различные идентификаторы для снижения уровня взаимных помех.

Вопросы частотного планирования в общем плане рассматриваются в ряде работ, однако в них отсутствуют конкретные методики и рекомендации по применению $[2,7,8]$.
В статье рассмотрены вопросы определения пространственно-технических параметров сотовой сети стандарта LTE на основе сложившихся подходов к решению задачи планирования сотовых систем мобильной связи и отмечена необходимость разработка методик:

построения частотных кластеров при заданной вероятности появления внутрисистемных помех, обеспечивающих максимизацию емкости кластерной структуры;

определения пространственных параметров сети, исходя из количества выделенных ресурсных блоков, распределения скоростей передачи, конфигурации оборудования и условий функционирования;

оценки средней пропускной способности и емкости сети для заданного профиля абонентского трафика и алгоритмов работы планировщиков;

частотного планирования и соответствующих рекомендаций по частотным разносам.

\section{СПИСОК ЛИТЕРАТУРЫ}

1. Бабков В.Ю., Вознюк М.А., Михайлов П.А. Сети мобильной связи. Частотнотерриториальное планирование. М.: Горячая линия - Телеком, 2007. 222 с.

2. Бабков В.Ю., Цикин И.А. Сотовые системы мобильной радиосвязи: Учеб. пособие. 2-е изд. перераб.и дополн. СПб.: БХВ - Петербург, 2013. $432 \mathrm{c}$.

3. 3GPP TS 36.104: Base Station (BS) radio transmission and reception. Apr. 2011. Release 9.

4. 3GPP TS 36.101: User Equipment (UE) radio transmission and reception. Nov. 2012. Release 11.

5. Рыжков А.Е., Воробьев В.О., Слышков
А.С., Сиверс М.А., Гусаров А.С., Шуньков Р.В. Стандарты и сети радиодоступа 4G. СПб.: Линк, 2012. $226 \mathrm{c}$.

6. Кошуняева Н.В., Патронова Н.Н. Теория массового обслуживания (практикум по решению задач). Архангельск: САФУ, 2013. 107 с.

7. Тихвинский В.О., Терентьев С.В., Высочин В.П. Сети мобильной связи LTE/LTE Advanced: технологии 4G, приложения и архитектура. М.: ИД «Медиа Паблишер», 2014. 384 с.

8. Salo J., Nur-Adam M., Chang K. Practical Introduction to LTE Radio Planning. Wiley and Son, 2010.

\section{REFERENCES}

1. Babkov V.Yu., Voznyuk M.A., Mikhaylov P.A. Seti mobilnoy svyazi. Chastotno-territorialnoye planirovaniye [Mobile network. Frequency planning]. Moscow: Goryachaya liniya - Telekom Publ., 2007, 222 p. (rus)

2. Babkov V.Yu., Tsikin I.A. Sotovyye sistemy mobilnoy radiosvyazi [Cellular mobile radio systems: $a$ tutorial]. St. Petersburg: BKhV - Petersburg Publ., 2013. 432 p. (rus)

3. 3GPP TS 36.104: Base Station (BS) radio transmission and reception, Release 9, April 2011.

4. 3GPP TS 36.101: User Equipment (UE) radio transmission and reception, Release 11, November 2012.

5. Ryzhkov A.Ye., Vorobyev V.O., Slyshkov A.S., Sivers M.A., Gusarov A.S., Shunkov R.V. Standarty $i$ seti radiodostupa $4 G$ [Standards and $4 G$ radio access network]. St. Petersburg: Link Publ., 2012, 226 p. (rus)

6. Koshunyayeva N.V., Patronova N.N. Teoriya massovogo obsluzhivaniya (praktikum po resheniyu zadach) [Queueing Theory (practical problem-solving)]. Arkhangelsk: SAFU Publ., 2013, 107 p. (rus)

7. Tikhvinskiy V.O., Terentyev S.V., Vysochin V.P. 
Seti mobilnoy svyazi LTE/LTE Advanced: tekhnologii $4 G$, prilozheniya $i$ arkhitektura [The mobile network LTE/LTE Advanced: technology 4G, applications and architecture]. Moscow: Media Publ.,
2014, 384 p. (rus)

8. Salo J., Nur-Adam M., Chang K. Practical Introduction to LTE Radio Planning. Wiley and Son, 2010.

БАБКОВ Валерий Юрьевич - профессор кафедры радиотехники и телекоммуникаций Санкт-Петербургского государственного политехнического университета, доктор технических наук.

195251, Россия, Санкт-Петербург, ул. Политехническая, д. 29.

E-mail: babkov_v@mail.ru

BABKOV, Valeriy Yu. St. Petersburg Polytechnic University.

195251, Politekhnicheskaya Str. 29, St. Petersburg, Russia.

E-mail: babkov_v@mail.ru

НИКИТИНА Александра Викторовна - доцент кафедры радиотехники и телекоммуникаций Санкт-Петербургского государственного политехнического университета, кандидат технических наук.

195251, Россия, Санкт-Петербург, ул. Политехническая, д. 29.

E-mail: envision@yandex.ru

NIKITINA, Aleksandra V. St. Petersburg Polytechnic University.

195251, Politekhnicheskaya Str. 29, St. Petersburg, Russia.

E-mail: envision@yandex.ru

СТАРИКОВ Владимир Владимирович - аспирант кафедры передающих устройств и средств подвижной связи Санкт-Петербургского государственного университета телекоммуникаций имени профессора М.А. Бонч-Бруевича.

191186, Россия, Санкт-Петербург, наб. р. Мойки, д. 61.

E-mail: vl.vl.starikov@gmail.com

STARIKOV, Vladimir V. Bonch-Bruevich Saint-Petersburg State University of Telecommunications.

191186, Moika Emb. 61, St. Petersburg, Russia.

E-mail: vl.vl.starikov@gmail.com 